\section{$\underset{\substack{\text { hommes } \\ \text { \& migrations }}}{ }$}

\section{Hommes \& migrations}

Revue française de référence sur les dynamiques

migratoires

1307 | 2014

L'Afrique qualifiée dans la mondialisation

\title{
Les étudiants maliens dans l'enseignement supérieur privé au Maroc
}

Enjeux et stratégies de distinction sociale par les études à l'étranger

\section{Niandou Touré}

\section{(2) OpenEdition}

12 Journals

\section{Édition électronique}

URL : http://journals.openedition.org/hommesmigrations/2874

DOI : 10.4000/hommesmigrations.2874

ISSN : 2262-3353

\section{Éditeur}

Musée national de l'histoire de l'immigration

\section{Édition imprimée}

Date de publication : 1 juillet 2014

Pagination : 29-36

ISBN : 978-2-919040-28-5

ISSN : 1142-852X

Référence électronique

Niandou Touré, "Les étudiants maliens dans l'enseignement supérieur privé au Maroc », Hommes \& migrations [En ligne], 1307 | 2014, mis en ligne le 01 juillet 2017, consulté le 01 mai 2019. URL : http:// journals.openedition.org/hommesmigrations/2874 ; DOI : 10.4000/hommesmigrations.2874 


\section{LES ÉTUDIANTS MALIENS DANS L'ENSEIGNEMENT SUPÉRIEUR PRIVÉ AU MAROC} ENJEUX ET STRATÉGIES DE DISTINCTION SOCIALE PAR LES ÉTUDES À L'ÉTRANGER

par NIANDOU TOURÉ, doctorant en sociologie à l'université Paris-Descartes.

Le développement accéléré de l'offre d'enseignement supérieur

privé au Maroc représente une nouvelle opportunité pour

les étudiants originaires d'Afrique subsaharienne. Grâce aux

accords bilatéraux organisant la mobilité estudiantine avec

leurs pays d'origine, ils viennent en nombre au Maroc chercher

une formation supérieure de qualité. L'obtention d'un titre

universitaire au Maroc, solution alternative à l'Europe, constitue

un capital social valorisé sur le marché malien de l'emploi.

La présence des étudiants maliens dans l'enseignement supérieur marocain remonte aux années $1960^{1}$. Le financement de la formation de ces premiers étudiants se faisait dans le cadre de programmes de renforcement des compétences mis en place, de façon ponctuelle, par l'État ou par certaines institutions spécialisées de l'Organisation des Nations Unies (Onu), dont l'Organisation mondiale de la santé (OMS).

Dans les décennies qui ont suivi, la formation des Maliens au Maroc est devenue un volet clé des accords de coopération bilatérale liant le Maroc à ses alliés au sud du Sahara : 1963 pour le Sénégal, 1972 pour le Gabon, 1973 pour la Côte d'Ivoire, 1977 pour le Nigeria et 1982 pour le Niger. Les bouleversements politiques des années 1980 admission de la République arabe sahraouie démocratique au sein de l'Organisation de l'unité africaine (OUA) en 1982, suivie du retrait définitif du Maroc de l'OUA en 1985 - vont conduire à redéfinir la politique extérieure du royaume chérifien, spécialement en direction du Sud. En effet, dès 1986, est créée l’Agence marocaine de coopération internationale (AMCI), bras armé de la politique d'influence du Maroc.

Ce dispositif du soft power marocain a notamment pour mission de gérer la coopération en matière de formation des cadres. Les accords dans ce domaine vont alors devenir systématiques à partir du milieu des années 1980 (avec le Mali et le Cameroun en 
1987, et une demi-douzaine d'autres entre 1988 et $\left.1997^{2}\right)$. Sélectionnés pour la plupart selon des critères d'excellence académique, les étudiants bénéficient d'une bourse d'études ${ }^{3}$ qui leur permet de s'inscrire dans les établissements publics du Maroc.

\section{Les mutations de l'enseignement supérieur marocain}

Chaque année, plusieurs centaines de Subsahariens, majoritairement francophones (Sénégalais, Maliens, Guinéens), mais pas uniquement (Mauritaniens, Angolais), débarquent dans le système marocain d'enseignement supérieur public afin de s'y former dans différentes disciplines, où les cours sont dispensés principalement en français. La mobilité pour études des étudiants d'Afrique noire au Maroc est une pratique courante depuis quelques décennies, avec la systématisation des accords bilatéraux. Cependant, elle connait de profondes mutations depuis une quinzaine d'années. La plus remarquable est la

Alors que la Tunisie semble s'être spécialisée dans l'accueil des étudiants du privé et que les étudiants étrangers

d'Algérie sont en grande

majorité des boursiers du public, le Maroc, lui, est chaque année l'hôte de plusieurs centaines d'étudiants inscrits dans les deux secteurs. tiaire comme un domaine complémentaire dans la formation et la recherche par son "innovation". Durant l'année universitaire 2012-2013, on dénombrait plus de deux cents écoles privées autorisées par le ministère de l'Enseignement supérieur ${ }^{4}$, elles jouent désormais un rôle déterminant dans la reconfiguration des dynamiques de mobilité étudiante Sud-Sud. Ces établissements accueillent une proportion très importante d'étudiants étrangers, dont les plus importants contingents sont originaires d'Afrique subsaharienne (Sénégal, Mali et Guinée en tête).

\section{Les raisons structurelles de l'attractivité du Maroc}

Le Maroc occupe une place particulière dans le paysage académique africain, non seulement en raison de sa mise en œuvre rapide de la réforme licencemaster-doctorat (LMD) par rapport à ses voisins du Maghreb, mais aussi parce qu'il a réussi la double ouverture internationale de ses écoles supérieures publiques et privées, que confirment toutes les statistiques. Alors que la Tunisie semble sêtre spécialisée dans l'accueil des étudiants du privé et que les étudiants étrangers d'Algérie sont en grande majorité des boursiers du public, le Maroc, lui, est chaque année l'hôte de plusieurs centaines d'étudiants inscrits dans les deux secteurs ${ }^{5}$. Il apparaît que les écoles privées recrutent un nombre important de leurs étudiants dans les pays coopérant avec le Maroc en matière de formation des cadres $^{6}$. Cela pose inévitablement la question de savoir comment la coopération bilatérale fait le lit de la nouvelle mobilité des étudiants dits "individuels" - c'est-à-dire qui ne sont pas boursiers. À travers un mécanisme de "causalité cumulative", la fréquentation massive du secteur public marocain d'enseignement supérieur a ainsi contribué à une plus grande visibilité du secteur privé dans les pays d'origine des étudiants étrangers du Maroc.

$\mathrm{Au}$ cours de notre recherche de thèse qui porte sur la mobilité sociale des Maliens par les études à létranger, nous avons réalisé des enquêtes qualitatives (entretiens semi-directifs et observation

2. Angola en 1998, Bénin en 1991, Burkina Faso et RDC en 1996, Tchad et Guinée en 1997. 3. Les Maliens perçoivent l'équivalent de $70 €$ par mois de l'AMCl et $40 €$ du Mali. 4. Source : http://www.enssup.gov.ma/doc_site/documents/Enseignement_superieur/prive/Liste_etab_ autorises.pdf. 5. Sylvie Mazzella (dir.), La Mondialisation étudiante. Le Maghreb éntre Nord et Sud, Paris, Khartala, 2009.

6. Johara Berriane, "La formation des élites subsahariennes au Maroc”, in Mansouria Mokhefi, Alain Antil (dir.), Le Maghreb et son Sud. Vers des liens renouvelés, Paris, CNRS, 2012. 
participante) dans les villes de Rabat, Casablanca et Fès. La ville de Fès, en particulier, présente des spécificités qui en font un intéressant terrain d'observation : on y trouve une importante population étudiante malienne, dont la grande majorité étudie dans le privé?. Par conséquent, on peut y analyser minutieusement les pratiques individuelles et collectives à l'œuvre dans ce qui a précédemment été décrit comme une libéralisation de l'enseignement supérieur ${ }^{8}$.

Partant de là, nous nous sommes interrogés sur l'ampleur de ce phénomène et de son impact sur les transformations des mobilités intra-africaines au début du XXIe siècle. Dans la présente contribution, nous proposons de répondre aux principales questions que soulève la situation des étudiants maliens au Maroc. Nous en avons relevé quatre, toutes liées. Tout d'abord, comment la demande d'éducation supérieure au Mali a trouvé une réponse dans l'enseignement supérieur privé marocain, au point de contribuer à l'essor de ce dernier? Quels sont les déterminants socio-économiques de la mobilité pour études des Maliens de l'enseignement supérieur privé marocain ? Quels sont les enjeux de distinction sociale sous-tendus par l'acquisition d'un titre universitaire à l'étranger dans le contexte malien ? Quelles stratégies individuelles ou collectives sont mobilisées aux fins de mobilité pour études?

\section{La complémentarité entre le public et le privé}

Les premières écoles supérieures privées du Maroc ont été créées à la fin des années 1980. Si on en retrouve plus de deux cents aujourd'hui, c'est la conséquence du boom consécutif à la loi 01-00, portant organisation de l'enseignement supérieur et promulguée en 2000 sous le gouverne- ment d'Abderrahman El Youssoufi. Cette loi, qui consacre trente articles à l'enseignement supérieur privé, le présente comme la réponse aux insuffisances de l'enseignement supérieur public, aussi bien en matière de formation que de recherche. L'analyse des plaquettes pédagogiques, des missions actuellement remplies par quelques écoles privées et leur répartition géographique sur le territoire marocain permettent de rendre compte de cette complémentarité. Par exemple, la distribution des filières d'enseignement est telle que la médecine reste encore Les écoles privées sont concentrées dans les grands centres urbains comme Casablanca, Rabat, Marrakech, Fès et Tanger, répondant à une demande croissante de formation. aujourd'hui quasi exclusivement réservée au domaine public. En revanche, des formations nouvelles telles que la biotechnologie et les spécialités comme le contrôle qualité tendent à devenir des filières de spécialisation des écoles privées comme l'École des hautes études en biotechnologie de Casablanca.

Les écoles privées sont concentrées dans les grands centres urbains comme Casablanca, Rabat, Marrakech, Fès et Tanger, répondant à une demande croissante de formation. L'augmentation de cette demande se justifie d'une part par le nombre important de diplômés de l'enseignement secondaire qui ne peuvent être accueillis dans les universités et autres établissements publics - notamment à cause d'une forte sélection à l'entrée dans certains cursus tels que la médecine ou les écoles d'ingénieur.

D'autre part, cette pression trouve son explication dans la demande de diversification de l'offre pédagogique de lécole publique, notamment del'université, jugée - par différents acteurs de l'école, au premier rang desquels les parents d'élèves - incompatible avec un marché de l'emploi en constante évolution. C'est à cette insuffisance de l'offre publique d'édu- 
Étudiants du département des arts et de la culture du campus universitaire de Cocody à Abidjan, mars 2010. o Camille Millerand

cation supérieure que prétend répondre la libéralisation de l'école. C'est ainsi que les écoles privées proposent des formations qui s'inscrivent essentiellement dans le domaine des sciences de l'entreprise (la gestion, le droit, le management, le marketing ou le commerce) ou des sciences et technologies (télécommunications, informatique, électronique, biotechnologies, etc.). Le système "dual" de l'école marocaine, hérité du système français, confère l'exclusivité des formations d'ingénieur et de commerce, en général, aux grandes écoles comme l'Institut national des postes et télécommunications (INTP), l'Institut agronomique et vétérinaire Hassan-II (IAV), l'Institut supérieur de commerce et d'administration des entreprises (ISCAE) ou l'École nationale de commerce et de gestion (ENCG). Face à la rigidité des processus d'orientation à l'issue du secondaire, les institutions privées opposent la souplesse : leur accès est souvent conditionné au seul versement des frais de scolarité. Et, en dépit de leurs coûts nettement plus importants que ceux de l'école publique, les écoles privées viennent combler les attentes de certaines catégories sociales de Maliens.

\section{Une panacée pour les classes aisées maliennes}

Les coûts de scolarité constatés auprès des étudiants maliens rencontrés dans le cadre de nos entretiens de recherche, réalisés en 2012 et 2013, 
sont compris entre 25000 et 60000 dirhams par an, l'équivalent de 2200 et 5500 euros. À cette somme, il faut ajouter le coût de la vie dans les grandes villes marocaines, afin d'avoir une idée des obligations financières des parents maliens qui supportent les frais de séjour de leurs enfants au Maroc. Par un croisement des informations recueillies auprès de nos 32 étudiants interviewés, nous sommes parvenu à estimer le coût moyen du séjour annuel entre 5000 à 9000 euros pour chaque étudiant. Ces sommes dépassent le niveau moyen de richesse économique au Mali, où le revenu national brut par habitant est seulement de 660 dollars par an ${ }^{9}$.

Ce grand contraste conduit à un questionnement sur les catégories sociales d'appartenance des étudiants maliens de l'enseignement supérieur privé au Maroc. S'il est évident que les parents de ces étudiants sont aisés, ils le sont, assez souvent, moins que les parents des étudiants "individuels" maliens du Canada, des États-Unis ou de la France, où nous avons également réalisé des terrains de recherche. Reste alors à les situer dans les classes supérieures ou moyennes du Mali.

La question des classes moyennes en Afrique fait l'objet de débats théoriques anciens et encore en cours dans le domaine de la recherche scientifique $^{10}$, cela est davantage vrai des pays les plus pauvres. Il n'en demeure pas moins pertinent de se questionner sur les catégories sociales dont sont issus les étudiants des écoles privées marocaines ; nous faisons le choix de prendre en considération les niveaux de revenus et les professions des parents des étudiants.

Nous avons adopté une méthode qualitative de recherche, en raison de l'absence de données sta- tistiques fiables ${ }^{11}$, nous privant d'une démarche scientifique soucieuse de la représentativité des filières d'enseignement, du genre, du niveau ou de la ville de formation. Il ressort des récits de vie recueillis ${ }^{12}$ que les étudiants sont en majorité issus de familles dans lesquelles au moins un des deux parents est instruit. Or ces derniers interviennent davantage que les parents des étudiants boursiers dans les choix d'orientation scolaire de leurs enfants. Ils sont cadres dans des ONG ou fonctionnaires de l'administration publique, exercent des professions libérales juridiques ou de santé, et sont plus rarement commerçants ou enseignants. Ces profils de parents sont assez comparables à ceux des étudiants maliens enquêtés en France. Le tiers de ces étudiants ont d'ailleurs un membre de leur fratrie immédiate dans un pays européen.

L'école privée marocaine est

S'il est évident que

les parents de ces étudiants sont aisés, ils le sont, assez souvent, moins que les parents des étudiants "individuels" maliens la réponse à des situations différentes. Pour certains, du Canada, des États-Unis ou de la France. la raison de ce choix tient au fait que, face à l'échec d'un premier projet d'études en direction de l'Europe, assez souvent la France, ils se rabattent sur l'option nord-africaine considérée comme la meilleure alternative à leur dessein initial. Mlle R., née en 1988 de parents enseignants du secondaire et du supérieur au Mali, est titulaire d'un diplôme d'ingénieur obtenu à Sup'Telecom Rabat et préparait, en 2012, un master en finance et stratégie dans une autre école privée de Rabat. Issue d'une famille imprégnée de la culture de la mobilité - ses quatre frères et quatre sœurs ont étudié en Chine, au Ghana, en France et 
aux États-Unis, où l'un d'eux s'est établi -, elle nous explique avoir prospecté les écoles tunisiennes et marocaines à défaut d'avoir un visa pour la France. Le choix du Maroc par rapport à la Tunisie, d'après elle, relèverait du pur hasard, mais serait un meilleur choix qu'une formation au Mali. Habituée des écoles privées dans lesquelles elle a fait l'essentiel de sa scolarité pré-universitaire au Mali, elle espère multiplier ses chances d'accès à l'emploi au Mali, grâce à ce passage à l'étranCertaines écoles ger qui lui paraît "naturel". privées marocaines bénéficient Pour d’autres - et ce cas d'une coopération avec est le plus récurrent dans des établissements supérieurs occidentaux, souvent en France et au Canada. notre échantillon -, qui n'ont pas tenté d'aller en Europe et n'ont pas candidaté aux programmes de bourses pour le Maghreb, mais qui disposent des moyens financiers requis, c'est une aubaine. D'après A., étudiant à l'Institut polytechnique privé de Casablanca: “J'aurais pu postuler pour une bourse, mais j'ai choisi de ne pas le faire. Et le Maroc, c'est proche de l'Europe, c'est presque pareil. En plus, il est possible dans mon école d'obtenir un diplôme équivalent de certaines universités canadiennes. Par exemple, nos cours sont les mêmes que ceux dispensés à l'université de Laval."

\section{Fès, ville universitaire "africaine" promue par des étudiants recruteurs}

Certaines écoles privées marocaines bénéficient d'une coopération avec des établissements supérieurs occidentaux, souvent en France et au Canada. C'est le cas notamment de trois écoles privées de la ville de Fès - Sup'management, l'École polytechnique supérieure d'informatique et électronique (EPSIEL) et les Hautes Études comptables et financières (HECF) -, dont les stratégies marketing, plutôt tournées vers l'Afrique, insistent sur leurs importants réseaux internationaux et la possibi- lité d'obtenir des diplômes français et canadiens en étant au Maroc. Les HECF ont un accord de partenariat avec l'ESC de Pau ; l'Epsiel avec l'université de Franche-Comté ; et Sup'management jouit d'un réseau mondial d'antennes et de collaborations en Espagne et aux États-Unis.

La communication des écoles privées passe d'abord par les réseaux d'interconnaissance : presque tous les étudiants interrogés ont expliqué avoir pris connaissance de l'existence de leur école par l'intermédiaire d'un proche. C'est de ce constat qu'est venue l'idée d'employer les étudiants étrangers comme relais dans leur pays de départ. Pour chacune de ces écoles, nous avons rencontré des étudiants maliens au Maroc, qui ont également une fonction, plus ou moins officielle, de recruteur.

S.-M a 20 ans et il est étudiant en $2^{e}$ année du cycle informatique et gestion de l'EPSIEL. Alors qu'il ne connaissait pas cette école avant d'y entrer - elle lui a été recommandée par une amie -, il a réussi à se faire désigner comme recruteur, après avoir proposé ses services à la direction de l'école dès la fin de sa première année. Contre une rémunération par étudiant inscrit qui varie entre 100 et $400 €$, le jeune homme s'active particulièrement sur les réseaux sociaux tels que Facebook afin de vendre au mieux les services de son école. Les deux dizaines d'étudiants qu'il a réussi à faire inscrire à l'école pour l'année universitaire 2013-2014 lui permettront d'alléger ses coûts de scolarité, estimés à $2500 €$, et de grossir les rangs des étudiants maliens qui représentent déjà un quart des effectifs de l'EPSIEL à Fès.

Lesétudiantsrecrutentdansleurscerclesimmédiats, puis ils vont au-delà. Le Maroc est donc devenu l'une des premières destinations pour études parce qu'il répond à la demande en formation - ou en titres - de populations africaines pas toujours assez riches pour aller en Europe ou en Amérique du Nord, mais pas non plus financièrement désarmées au point de se former dans les établissements locaux qu'elles estiment sur le déclin. 


\section{Les déterminants sociaux de la mobilité des Maliens}

La mobilité sortante pour études, au départ du Mali, est une pratique remontant, pour l'école moderne, à la période coloniale. En effet, les écoles de l'Afrique-Occidentale française, à l'instar de l'École normale William-Ponty, ont constitué les premiers cadres de l'instruction dans la sous-région ${ }^{13}$. Obtenu à l'étranger ou au Mali, le diplôme a, avant tout, une fonction sociale d'ascension ou de pérennisation d'un rang. La relation entre formation et emploi, et donc statut social, était quasiment spontanée du temps où les diplômés trouvaient facilement du travail. Les changements structurels de léconomie malienne au début des années 1980 ont conduit à des réformes telles que l'institution d'un concours d'entrée à la fonction publique, et, très rapidement, sont apparus des diplômés sans emploi ${ }^{14}$. L'effritement du système scolaire depuis une vingtaine d'années et la rupture de la passerelle entre formation et emploi public rémunéré ont érigé la mobilité internationale pour études en une nécessité pour ceux qui en ont les moyens.

Pour les étudiants individuels et leurs parents avec qui ils conçoivent le projet de mobilité, le choix de la formation à l'étranger comporte d'abord des enjeux de démarcation par rapport aux autres diplômés d'un marché de l'emploi qui fait de moins en moins confiance aux diplômés locaux. Il s'agit donc d'acquérir un label "diplôme marocain", auquel on attribue des qualités supérieures aux grades universitaires délivrés sur place. Cette recherche d'un label étranger est confirmée par l'importante présence de Maliens, de Mauritaniens ou de Sénégalais à Sup'management Maroc, alors que des antennes sont présentes à Bamako, Nouadhibou et Dakar. L'observation de la scolarité antérieure au Maroc et l'analyse à l'échelle des cellules familiales et des itinéraires d'études des fratries de nos enquêtés ont permis de déterminer le poids des caractéristiques sociales des étudiants dans l'investissement plus ou moins important des familles.

\section{Des stratégies familiales de distinction sociale}

La quasi-totalité des étudiants du privé que nous avons rencontrés, 27 sur 32, ont obtenu leur dernier diplôme malien dans une école privée de Bamako. Le détail de leur cursus scolaire antérieur fait ressortir des parcours caractérisés par une prédominance de lécole privée. L'orientation scolaire est donc stratégique pour ces étudiants depuis le début de leur scolarité. Ce choix de l'école privée s'explique d'abord par la confiance que les parents placent dans ce système, en

réaction aux insuffisances selon la structure des d'une école publique dont les familles, les plans familiaux différents acteurs décrient les de scolarité diffèrent tares multiples, notamment suivant le genre à cause du désengagement et l'ordre de l'étudiant(e) des autorités. Quant à la pré- dans la fratrie. sence importante d'étudiants ressortissants de la capitale malienne, elle se justifie par les canaux de communication des écoles qui se font dans des réseaux d'interconnaissance des étudiants, et par la forte concentration de couches moyennes supérieures dans l'agglomération qui compte un septième des Maliens ${ }^{15}$.

Selon la structure des familles, les plans familiaux de scolarité diffèrent suivant le genre et l'ordre de l'étudiant(e) dans la fratrie. Ainsi, O., étudiant en droit à Mohammedia, est le troisième enfant d'une fratrie de cinq enfants. Il nous explique que ses sœurs aînées sont restées au Mali pour leurs études supérieures. Dans ce cas, la mobilité à 
l'étranger est à la fois un sacrifice pour (et de) l'enfant. Quant à F., étudiant à Rabat, dont les deux sœurs étudient en France, il est au Maroc parce que les couts de la formation en France sont trop élevés dans les grandes écoles pour que ses parents y envoient trois enfants. Il paie alors pour être le dernier, et peut-être aussi parce qu'il est de sexe masculin.

Pendant leurs études et après, les étudiants maliens des écoles supérieures privées marocaines bénéficient des réseaux de leur école et de leur famille, qui leur permettent de réaliser leurs stages dans des établissements privés au Maroc ou au Mali. En dépit des critiques formulées à l'encontre des quelques écoles qu'on taxe de vendre les diplômes, la reproduction sociale, qui est l'objectif central des stratégies de démarcation, est réussie. Ladite réussite peut s'observer dans les trajectoires de retour des diplômés maliens formés au Maroc. En raison de la législation marocaine en matière d'intégration du marché du travail, les diplômés sénégalais sont les seuls habilités à y travailler dans l'ensemble des domaines de professionnalisation. Quant aux autres, dont les Maliens, ils se voient opposer une préférence nationale, rendant leur insertion plus compliquée dans le royaume. Cette réalité amenuise le nombre d'installations dans le pays, ce qui contraste grandement avec la présence des diplômés maliens en France, notamment.

\section{Conclusion}

Le nombre d'étudiants maliens au Maroc augmente de plusieurs centaines chaque année - cent boursiers bénéficiant de l'allocation de l'Agence marocaine de coopération internationale (AMCI) et de celle du Mali, un nombre variable de boursiers dits "hors quotas" et des effectifs encore moins saisissables d'étudiants du privé. Ce sont pourtant ces derniers qui représentent la plus grande cohorte d'étudiants à chaque rentrée universitaire. Le cas malien s'explique par l'échec relatif du système d'enseignement supérieur local et démontre comment l'école privée peut pallier le besoin de qualification dans le contexte d'un système éducatif perçu par ses propres acteurs comme défaillant. L'essor de l'école privée marocaine répond non seulement à des besoins internes d'offre d'éducation supérieure en colmatant certaines brèches de l'enseignement public, mais permet également de répondre aux attentes des étudiants subsahariens dont les universités nationales sont en échec. Bien plus qu'une solution ponctuelle, la mobilité des Maliens dans les écoles supérieures privées du Maroc est une réponse à des quêtes diverses : accès au savoir, enseignement de meilleure qualité, mais aussi et surtout acquisition d'un titre universitaire valorisé sur le marché malien de l'emploi. Le capital social jouant ensuite, la réinsertion des étudiants se passe assez aisément une fois qu'ils sont de retour au pays.

Pour beaucoup de ces étudiants, le Maroc n'est ni un strapontin vers l'Europe, ni un choix contraint. Il répond à leurs aspirations et correspond aux moyens financiers qu'ils sont prêts à consentir pour se former. Ces coûts font rejaillir le sempiternel débat sur la libéralisation de l'éducation et les dérives potentielles de marchandisation des titres universitaires au détriment du savoir. Nos observations de terrain nous invitent à tempérer les critiques: les étudiants dans l'enseignement supérieur privé marocain sont bien des clients, acheteurs d'un savoir et d'un titre universitaire, mais ce sont également des apprenants pour qui l'enjeu est aussi de "se maintenir", de garder un rang social qui commence par l'obtention d'un travail après leur retour. 